\title{
Zoledronic acid enhances antitumor efficacy of liposomal doxorubicin
}

\author{
YOSHIYUKI HATTORI $^{1}$, KAZUHIKO SHIBUYA ${ }^{1}$, KAORI KOJIMA $^{1}$, ANDANG MIATMOKO ${ }^{1}$, \\ KUMI KAWANO $^{1}$, KEI-ICHI OZAKI ${ }^{2}$ and ETSUO YONEMOCHI ${ }^{1}$ \\ ${ }^{1}$ Institute of Medicinal Chemistry, Hoshi University, Shinagawa-ku, Tokyo 142-8501; \\ ${ }^{2}$ Laboratory of Cell Regulation, Department of Pharmaceutical Sciences, \\ Graduate School of Biomedical Sciences, Nagasaki University, Nagasaki 852-8521, Japan
}

Received February 20, 2015; Accepted April 2, 2015

DOI: 10.3892/ijo.2015.2991

\begin{abstract}
Previously, we found that the injection of zoledronic acid (ZOL) into mice bearing tumor induced changes of the vascular structure in the tumor. In this study, we examined whether ZOL treatment could decrease interstitial fluid pressure (IFP) via change of tumor vasculature, and enhance the antitumor efficacy of liposomal doxorubicin $\left(\right.$ Doxil $\left.^{\circledR}\right)$. When ZOL solution was injected at $40 \mu \mathrm{g} / \mathrm{mouse}$ per day for three consecutive days into mice bearing murine Lewis lung carcinoma LLC tumor, depletion of macrophages in tumor tissue and decreased density of tumor vasculature were observed. Furthermore, ZOL treatments induced inflammatory cytokines such as interleukin (IL)-10 and -12 , granulocyte-macrophage colony-stimulating factor (GM-CSF) and tumor necrosis factor (TNF)- $\alpha$ in serum of LLC tumor-bearing mice, but not in normal mice, indicating that ZOL treatments might induce an inflammatory response in tumor tissue. Furthermore, ZOL treatments increased antitumor activity by Doxil in mice bearing a subcutaneous LLC tumor, although they did not significantly increase the tumor accumulation of doxorubicin (DXR). These results suggest that ZOL treatments might increase the therapeutic efficacy of Doxil via improvement of DXR distribution in a tumor by changing the tumor vasculature. ZOL treatment can be an alternative approach to increase the antitumor effect of liposomal drugs.
\end{abstract}

\section{Introduction}

Normal blood vessels are organized in a hierarchy of evenly distributed arteries, capillaries and veins. The vessels are

Correspondence to: Dr Yoshiyuki Hattori, Institute of Medicinal Chemistry, Hoshi University, Ebara 2-4-41, Shinagawa-ku, Tokyo 142-8501, Japan

E-mail: yhattori@hoshi.ac.jp

Key words: zoledronic acid, doxorubicin, liposome, tumor-associated macrophage covered by pericytes to maintain vascular integrity (1). Unlike normal blood vessels, tumor vessels are structurally and functionally abnormal. Tumor blood vessels are absent in pericyte coverage, and are highly permeable to plasma and plasma proteins. An imbalance of pro- and anti-angiogenic factors causes endothelial cell migration and proliferation (2). It is well known that vascular endothelial growth factor (VEGF) plays an important role in tumor neovascularization.

Tumor-associated macrophages (TAMs) are abundant immunosuppressive cells recruited into the tumor microenvironment by cytokines such as macrophage colony-stimulating factor (M-CSF). The relevance of TAMs to tumor progression and metastasis is well established, and they promote angiogenesis, tissue remodeling and repair $(3,4)$. TAMs have the potential to release angiogenic growth factors such as VEGF and thereby enhance the formation of tumor vasculature $(5,6)$. Therefore, TAMs are potential targets for anticancer and antiangiogenic therapy.

Bisphosphonates such as clodronic acid and zoledronic acid (ZOL) are compounds used to prevent or inhibit the development of bone metastasis or excessive bone resorption and for the therapy of inflammatory diseases such as rheumatoid arthritis and osteoarthritis $(7,8)$. ZOL is a highly charged hydrophilic molecule that does not readily cross the plasma cell membrane, and it reaches pharmacologically active concentrations only in cells that exhibit marked fluid-phase endocytosis, such as osteoclasts and macrophages. Therefore, ZOL is an efficient reagent for the selective depletion of macrophages. The use of ZOL as an anti-angiogenic agent has been found to suppress solid tumor growth (9).

Anti-angiogenesis effects are known to change the tumor vasculature. Preclinical studies have shown that anti-VEGF therapy changes the tumor vasculature toward a more mature or normal phenotype (10). Normalization of disorganized tumor vasculature using therapeutics, rather than the blockage or disruption of tumor blood vessels, reduces tumor hypoxia, interstitial fluid pressure (IFP) and hyper-permeability and facilitates the delivery of exogenous therapeutics. Therefore, tumor vascular normalization has become a complementary therapeutic paradigm for cancer $(1,2)$. The anti-angiogenesis effect has already been applied in combination therapy. Bevacizumab, an anti-VEGF antibody, was developed for 
blocking angiogenesis, and it is used clinically with other drugs to improve the efficiency of conventional chemotherapy.

Previously, we found that intravenous injections of ZOL solution into tumor-bearing mice induced changes of vascular structure in the tumor (11); however, the effect of ZOL on the tumor microenvironment was not clear. Polyethylene glycol (PEG)-modified liposomes are long-lived in the circulation and accumulate passively in tumors. The tumor accumulation of the liposomes in tumor tissues is due to leakiness of tumor vessels to the macromolecular agents [enhanced permeability and retention (EPR) effect]. It has been reported that transforming growth factor (TGF)- $\beta$ type I receptor inhibitors were able to increase the antitumor effect of liposomal DXR or micelle DXR by changing the microenvironment of the vasculature $(12,13)$. Therefore, in this study, we examined whether ZOL treatments could facilitate the delivery of liposomal DXR (Doxil) by changing the microenvironment of the vasculature and increase therapeutic efficacy in vivo.

\section{Materials and methods}

Materials. Zoledronic acid (ZOL) was obtained from Enzo Life Sciences (Farmingdale, NY, USA). Doxorubicin hydrochloride (DXR) was purchased from Wako Pure Chemical Industries Inc. (Osaka, Japan). Liposomal DXR, Doxil, was obtained from Janssen Pharmaceutical K.K. (Tokyo, Japan). All other chemicals were of the finest grade available.

Cell culture. Murine Lewis lung carcinoma LLC was obtained from the Cell Resource Center for Biomedical Research, Tohoku University (Miyagi, Japan). Murine macrophage RAW264.7 was obtained from the European Collection of Cell Cultures (ECACC, Wiltshire, UK). LLC and RAW264.7 cells were cultured in RPMI-1640 medium with $10 \%$ heat-inactivated fetal bovine serum (FBS) and kanamycin $(100 \mu \mathrm{g} / \mathrm{ml})$ in a humidified atmosphere containing $5 \% \mathrm{CO}_{2}$ at $37^{\circ} \mathrm{C}$.

Tumor model. All animal experiments were performed with approval from the Institutional Animal Care and Use Committee of Hoshi University. For the generation of LLC tumors, $1 \times 10^{6}$ cells suspended in $100 \mu$ l of PBS were inoculated subcutaneously into the flank of female C57BL/6N mice (Sankyo Lab. Service Corp.). The tumor volume was calculated using the following formula: tumor volume $=0.5 \mathrm{x} \mathrm{a} \mathrm{x} \mathrm{b}^{2}$, where $\mathrm{a}$ and $\mathrm{b}$ are the larger and smaller diameters, respectively.

Immunohistochemical analysis. To examine the anti-angiogenic effect of ZOL on tumor, we intravenously injected ZOL solution at a dose of 5, 20 or $40 \mu \mathrm{g}$ of $\mathrm{ZOL} / \mathrm{mouse}$ per day for one, two or three consecutive days into mice bearing an LLC tumor when the tumor volume reached $\sim 200 \mathrm{~mm}^{3}$. The tumors $24 \mathrm{~h}$ after the final injection of ZOL solution were frozen on dry ice and sliced at $16 \mu \mathrm{m}$. Their sections were incubated with rat anti-mouse CD31 (PECAM-1) monoclonal antibody (Clone MEC 13.3, BD Pharmingen, San Diego, CA, USA) for the detection of mouse endothelial cells, and subsequently incubated with goat anti-rat IgG conjugated to Alexa Fluor 488 (Invitrogen, Carlsbad, CA, USA) as a secondary antibody. In the detection of mouse pericytes, the sections were further incubated with $\mathrm{Cy} 3$-conjugated rabbit anti-smooth muscle $\alpha$-actin ( $\alpha$-SMA) antibody (Sigma-Aldrich, MO, USA).

To examine the effect of ZOL on macrophages in tumor and liver, we intravenously injected ZOL solution at a dose of $40 \mu \mathrm{g}$ of $\mathrm{ZOL} / \mathrm{mouse}$ per day for three consecutive days into mice bearing an LLC tumor. The sections of tumor and liver $24 \mathrm{~h}$ after the final injection of ZOL solution were incubated with rat anti-mouse F4/80 monoclonal antibody (Clone CI:A3-1, AbD Serotec, Oxford, UK) for the detection of mouse macrophages, and subsequently incubated with goat anti-rat IgG conjugated to Alexa Fluor 488 as a secondary antibody. Immunofluorescence was examined microscopically using an Eclipse TS100-F microscope (Nikon, Tokyo, Japan).

IFP measurement in tumors. When the tumor volume reached $\sim 150 \mathrm{~mm}^{3}$, the LLC tumor-bearing mice were intravenously injected with ZOL solution at a dose of 5, 20 or $40 \mu \mathrm{g}$ of ZOL/ mouse per day for three consecutive days. Twenty-four hours after the final injection of ZOL solution, the mice were anesthetized with isoflurane, and then interstitial fluid pressure (IFP) of tumors was measured with a needle probe pressure monitor, fitted with an 18-gauge side-ported needle (Intra-Compartmental Pressure Monitor System; Stryker, Kalamazoo, MI, USA) connected to a syringe filled with $0.9 \%$ saline, as previously reported (14). The needle probe was inserted into the center of the tumor or normal muscle, and IFP was recorded. The IFP in tumors was normalized to that in muscle [normalized IFP = IFP (mmHg) of tumor/ IFP (mmHg) of muscle].

Determination of serum cytokine levels. When the tumor volume reached $\sim 150 \mathrm{~mm}^{3}$, LLC tumor-bearing mice were intravenously injected with ZOL solution at a dose of $40 \mu \mathrm{g}$ of $\mathrm{ZOL} / \mathrm{mouse}$ per day for three consecutive days. Twenty-four hours after the final injection of ZOL solution, serum was prepared by separation of the coagulated whole blood. Serum cytokine levels, including interleukin (IL)-10 and -12 (p70), granulocyte-macrophage colony-stimulating factor (GM-CSF) and tumor necrosis factor (TNF)- $\alpha$, were determined using mouse cytokine Th1/Th2 Panel (Bio-Rad, Hercules, CA, USA) and Bio-Plex 200 system (Bio-Rad). Normal values were determined using blood obtained from age-matched, normal mice without an LLC tumor.

Quantitative real-time PCR. When the tumor volume reached $\sim 200 \mathrm{~mm}^{3}$, the LLC tumor-bearing mice were intravenously injected with ZOL solution at a dose of $40 \mu \mathrm{g}$ of ZOL/mouse per day for three consecutive days. For the expression level of vascular endothelial growth factor (VEGF) mRNA in tumor tissues, the tumors were excised from LLC tumor-bearing mice $24 \mathrm{~h}$ after the final injection of $\mathrm{ZOL}$ solution, and then total RNA was isolated from the tumors using the TRI Reagent (Molecular Research Center, Inc., Cincinnati, OH, USA). RNA yield and purity were checked by spectrometric measurements at 260 and $280 \mathrm{~nm}$. cDNA was synthesized from total RNA by using the PrimeScript RT Reagent kit with gDNA Eraser (Takara Bio Inc., Shiga, Japan). Quantitative real-time PCR was performed with the Takara Thermal Cycler Dice (Takara Bio Inc.) and TaqMan Gene expression assays (vegfa: Mm00437306_m1, gapdh: Mm99999915_g1; Applied 
Biosystems, CA, USA). Samples were run in triplicate and the expression levels of VEGF mRNA were normalized for the amount of glyceraldehyde-3-phosphate dehydrogenase (GAPDH) mRNA in the same sample, and analyzed using the comparative $\mathrm{Ct}$ method.

Cytotoxicity. LLC and RAW264.7 cells were seeded separately at a density of $1 \times 10^{4}$ cells per well in 96-well plates and maintained in RPMI-1640 medium supplemented with 10\% FBS for $24 \mathrm{~h}$ before treatment. To examine cytotoxicity for ZOL, LLC and RAW 264.7 cells were treated with medium containing a range of 2.5 to $40 \mu \mathrm{M} \mathrm{ZOL}$, and they were then incubated for $48 \mathrm{~h}$. To examine the effect of ZOL on the cytotoxicity of DXR, LLC and RAW 264.7 cells were treated with medium containing a range of 0.125 to $2 \mu \mathrm{M}$ DXR in the presence or absence of $20 \mu \mathrm{M} \mathrm{ZOL}$ and they were then incubated for $48 \mathrm{~h}$. The cell number was determined with Cell Counting Kit-8 (Dojindo Laboratories, Kumamoto, Japan). Cell viability is expressed relative to the absorbance at $450 \mathrm{~nm}$ of untreated cells.

In vivo therapeutic studies. When the average volume of the tumors reached $100-200 \mathrm{~mm}^{3}$ in mice bearing LLC tumors, ZOL solution was intravenously administered via lateral tail veins at a dose of $40 \mu \mathrm{g}$ of $\mathrm{ZOL} /$ mouse on days 0,1 and 2, and then Doxil was intravenously administered at a dose of $5 \mathrm{mg}$ of DXR/kg on day 3. Tumor volume and body weight were measured for individual animals.

Biodistribution of DXR. When the average volume of the tumors reached $150 \mathrm{~mm}^{3}$ in mice bearing LLC tumors, ZOL solution was intravenously administered via lateral tail veins at a dose of $40 \mu \mathrm{g}$ of $\mathrm{ZOL} /$ mouse on days 0,1 and 2, and then Doxil was intravenously administered at a dose of $5 \mathrm{mg}$ of $\mathrm{DXR} / \mathrm{kg}$ on day 3 . The tumors and organs were excised $24 \mathrm{~h}$ after the injection of Doxil, and then homogenized in $0.1 \mathrm{M}$ $\mathrm{NH}_{4} \mathrm{Cl} / \mathrm{NH}_{3}$ buffer ( $\mathrm{pH} 9.0$ ). DXR was extracted with chloroform/methanol (2:1 v/v) and analyzed by HPLC, as previously described (13).

Statistical analysis. The statistical significance of differences between mean values was determined by Student's t-test. Multiple measurement comparisons were performed by analysis of variance followed by one-way analysis of variance on ranks with post hoc Tukey-Kramer's test. A p-value of $\leq 0.05$ was considered significant.

\section{Results}

Vascular structure of tumor after treatment with ZOL. Previously, we reported that the change of vascular structure in tumor was observed when ZOL solution was intravenously injected into tumor-bearing mice (11); however, the change of tumor environment upon ZOL treatments was not clear. In this study, we investigated whether ZOL treatments could improve the tumor environment via change of tumor vasculature and enhance the antitumor efficacy of liposomal DXR, Doxil.

First, to examine the frequency of ZOL administration and dosage amount $(\mu \mathrm{g})$ of $\mathrm{ZOL}$ required to change the vascular structure in LLC tumor, we intravenously injected ZOL solu- tion at a dose of $40 \mu \mathrm{g}$ of $\mathrm{ZOL} /$ mouse per day for one, two or three consecutive days into mice bearing an LLC tumor. When ZOL was injected for three consecutive days, apparent changes of vascular structure in the tumor were observed by immunostaining for CD31, which is a marker for endothelial cells, compared with those after one or two administrations (Fig. 1A). Regarding dosage amount, changes of vascular structure in the tumor were observed upon $\mathrm{ZOL}$ injection at 5, 20 and $40 \mu \mathrm{g}$ of $\mathrm{ZOL} /$ mouse per day for three consecutive days (Fig. 1B). ZOL treatments reduced narrow vessels in tumor and increased open vessels, indicating that blood flow in the tumor might be improved by the change of vasculature structure. Furthermore, some CD31-positive endothelial cells were covered with $\alpha$-SMA-positive pericytes in tumor section treated at $40 \mu \mathrm{g}$ of $\mathrm{ZOL} /$ mouse for three consecutive days, although most of the CD31-positive endothelial cells in tumor section of untreated mouse were not covered with $\alpha$-SMApositive pericytes (Fig. 1C), suggesting that ZOL treatments did not markedly affect pericyte coverage in tumor vessels. This histological change of tumor vasculature after ZOL treatment seemed to be similar to the phenomenon called normalization of the tumor vasculature (10).

Change of IFP. To examine the effect of ZOL on IFP in tumors, we measured IFP of tumors and muscles $24 \mathrm{~h}$ after intravenous injections of ZOL, and normalized the IFP of tumors by that of muscles. When ZOL solution was injected for three consecutive days, normalized IFP in LLC tumors was significantly decreased by injections of ZOL solution at 20 and $40 \mu \mathrm{g}$ of ZOL/ mouse per day $(5.8 \pm 1.3$ and $5.9 \pm 1.3$ in normalized IFP, respectively) compared with no treatment $(9.1 \pm 1.4$ in normalized IFP), but not by $5 \mu \mathrm{g}$ of $\mathrm{ZOL} /$ mouse per day $(6.6 \pm 2.6$ in normalized IFP) (Fig. 2). This indicated that the injection of 20 or $40 \mu \mathrm{g}$ of ZOL could decrease the IFP of the tumor by changing the tumor vasculature. Therefore, in subsequent experiments, we performed injections of $40 \mu \mathrm{g}$ of $\mathrm{ZOL} /$ mouse per day for three consecutive days.

It has been reported that Colon 26 and LLC tumors have well- and poorly vascularized blood vessels, respectively (15). Previously, we reported that LLC tumors showed higher IFP than Colon 26 tumors (14). When ZOL solution was injected into mice bearing Colon 26 tumor for three consecutive days, no decrease of IFP in the tumor was observed $(2.4 \pm 1.0$ and $3.7 \pm 1.2$ of normalized IFP in Colon 26 tumor with no treatment and ZOL treatment, respectively) (data not shown). These findings suggest that reduction of IFP in tumor by ZOL treatments might be effective for tumors having high IFP.

Change of macrophages in tumor and cytokine levels in serum after ZOL treatments. Bisphosphonates are internalized into cells by fluid-phase endocytosis, and then endosomal acidification causes the release of the bisphosphonates into the cytosol (16). Highly phagocytic cells such as macrophages have the ability to internalize bisphosphonates, which makes them an ideal target for these drugs. Therefore, we examined the effect of ZOL on macrophages in tumor and liver. In untreated mice, a large number of macrophages in the livers and tumors was detected by immunostaining with F4/80 antibody; however, in ZOL treatments, the number of macrophages in the tumors and livers was markedly decreased (Fig. 3). 
A

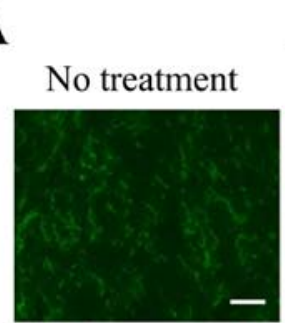

Number of injections ( $40 \mu \mathrm{g} \mathrm{ZOL} /$ mouse)
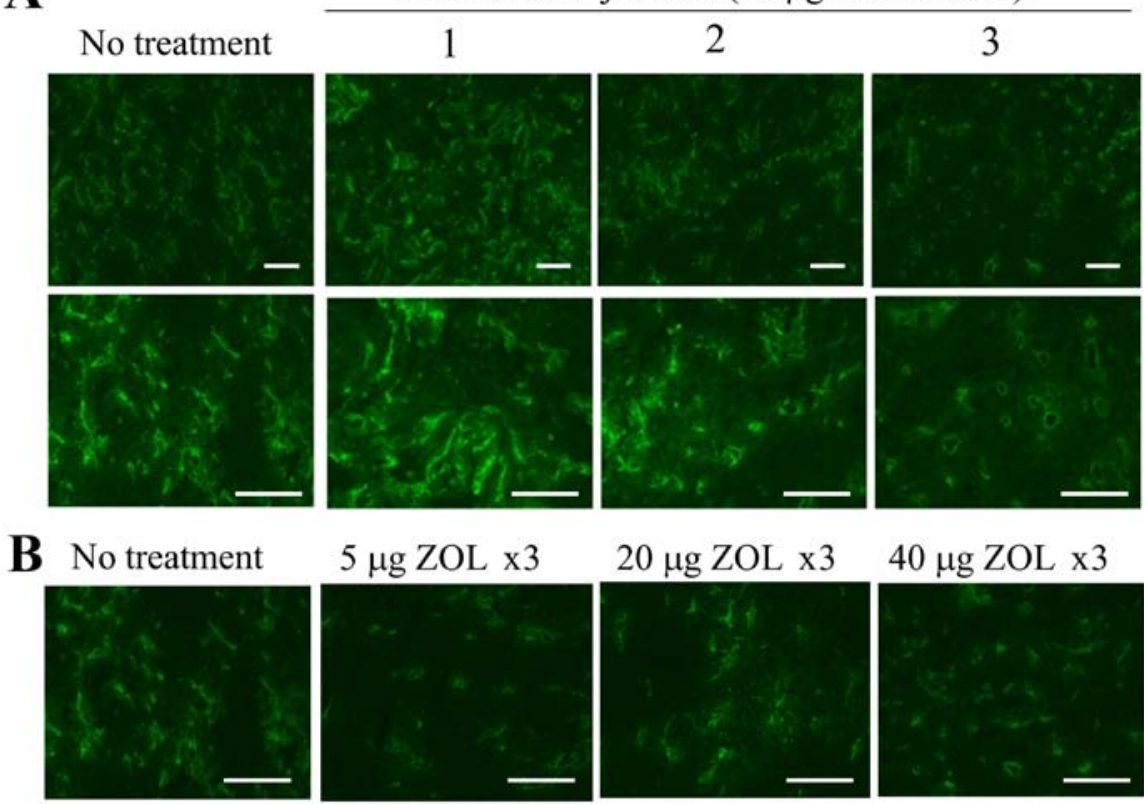

$5 \mu \mathrm{g} \mathrm{ZOL} \mathrm{x3}$

$20 \mu \mathrm{g} \mathrm{ZOL} \mathrm{x3}$

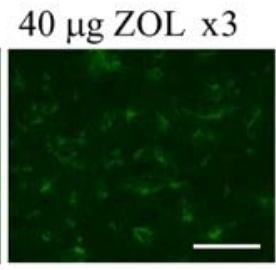

C
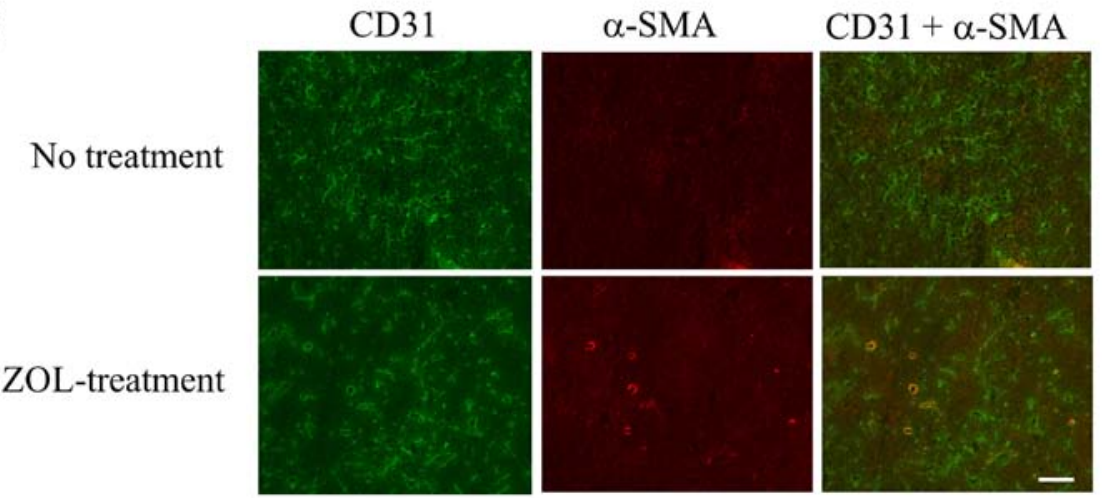

Figure 1. Immunostaining for endothelial cells after injection of ZOL into LLC tumor-bearing mice. Green signals indicate CD31-positive endothelial cells. (A) ZOL solution was intravenously injected at a dose of $40 \mu \mathrm{g}$ of ZOL/mouse per day into tumor-bearing mice for one, two or three consecutive days. (B) ZOL solution was intravenously injected at a dose of 5, 20 or $40 \mu \mathrm{g}$ of ZOL/mouse per day into tumor-bearing mice for three consecutive days. (C) ZOL solution was intravenously injected at a dose of $40 \mu \mathrm{g}$ of ZOL/mouse per day into tumor-bearing mice for three consecutive days, and immunostaining for endothelial cells and pericytes was performed. Green signals indicate CD31-positive endothelial cells and $\alpha$-SMA-positive red signals indicate pericytes. Scale bar, $100 \mu \mathrm{m}$.

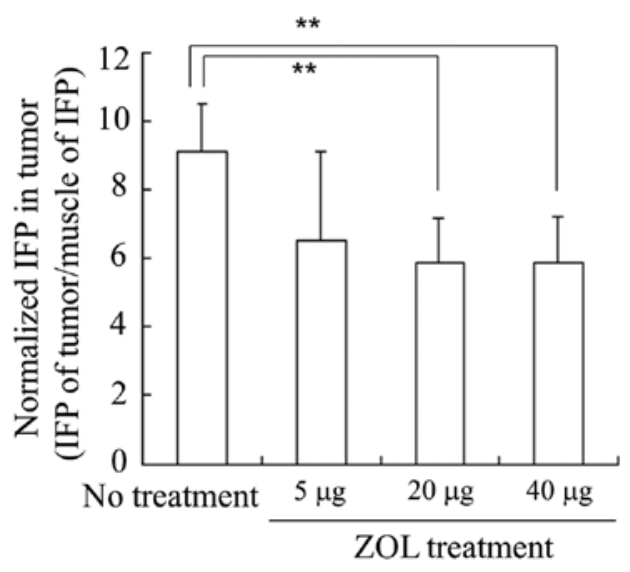

Figure 2. Change of IFP in LLC tumors after intravenous injection of ZOL. IFP in tumors was measured $24 \mathrm{~h}$ after intravenous injection of ZOL solution at a dose of 5,20 or $40 \mu \mathrm{g}$ of $\mathrm{ZOL} /$ mouse per day for three consecutive days into tumor-bearing mice. Normalized IFP of tumors was calculated by dividing IFP of tumors $(\mathrm{mmHg})$ by IFP of normal muscle $(\mathrm{mmHg})$. Each column represents the mean \pm SD $(n=4-5)$. Statistical significance was evaluated by Student's t-test. ${ }^{* *} \mathrm{p}<0.01$, compared with no treatment.
To examine the effect of ZOL treatments on the inflammatory cytokines in serum, we measured IL-10, IL-12 (p70), GM-CSF and TNF- $\alpha$ levels in serum after ZOL treatment in mice with or without LLC tumors. The ZOL injections into mice without tumor decreased IL-12, GM-CSF and TNF- $\alpha$ levels compared with those in untreated mice without tumor, although their levels were not significantly different (Fig. 4A). In contrast, the ZOL injections into LLC tumor-bearing mice significantly increased IL-12 and TNF- $\alpha$ levels compared with those of untreated mice bearing LLC tumors. These findings suggest that ZOL injections may affect tumor cells or TAMs in tumor tissues and induce inflammatory responses.

The vascular endothelial growth factor (VEGF) protein is a prominent cytokine, which promotes endothelial cell proliferation during angiogenesis. Therefore, we investigated whether ZOL treatments could affect the expression level of VEGF mRNA in the tumor by quantitative RT-PCR analysis. Surprisingly, VEGF mRNA level was not changed by ZOL treatments (Fig. 4B), indicating that the change of vascular structure might be caused in a VEGF-independent manner. 

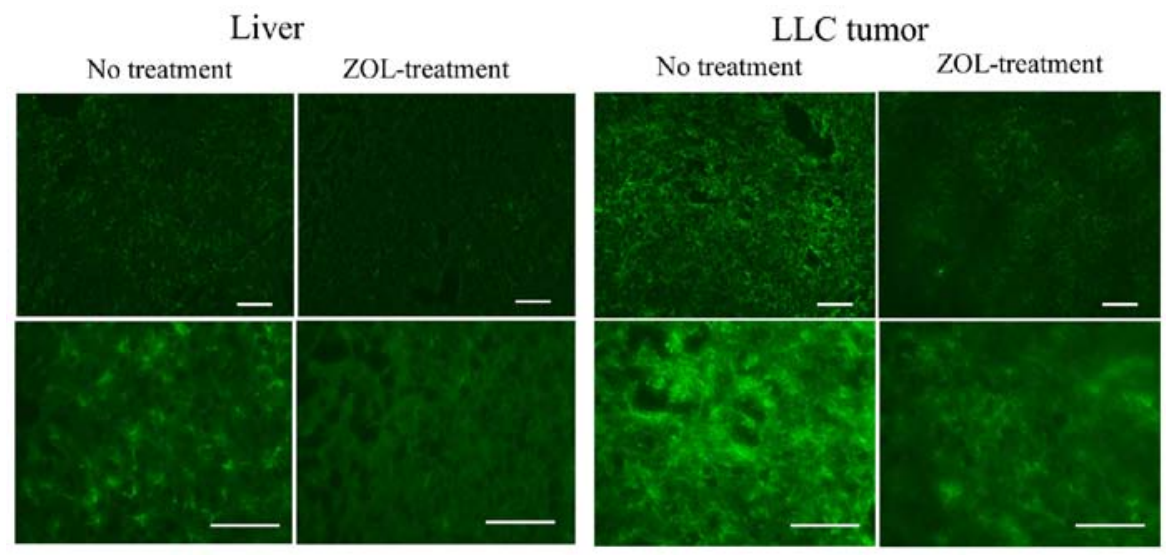

Figure 3. Immunostaining for macrophages in LLC tumor and liver after injection of ZOL into LLC tumor-bearing mice. ZOL solution was intravenously injected into mice bearing a tumor at a dose of $40 \mu \mathrm{g}$ of $\mathrm{ZOL} /$ mouse per day for three consecutive days. Green signals indicate F4/80-positive macrophage cells. Scale bar, $100 \mu \mathrm{m}$.

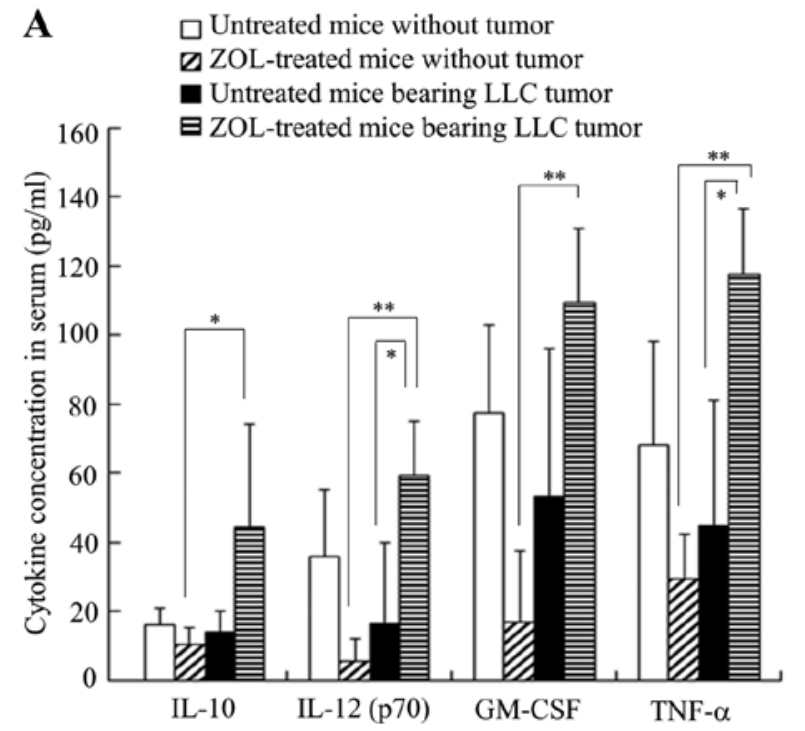

B VEGF mRNA

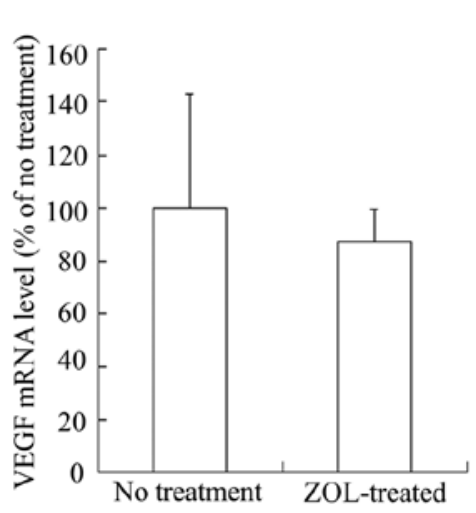

Figure 4. Cytokine levels in serum (A) and VEGF mRNA level (B) after ZOL injections. Mice with or without LLC tumors were intravenously injected with ZOL solution at a dose of $40 \mu \mathrm{g}$ of ZOL/mouse per day for three consecutive days. (A) Twenty-four hours after the final injection of ZOL solution, cytokine levels in the serum were determined. Each column represents the mean $\pm S D(n=3-4)$. The significance of differences between different groups was analyzed by one-way analysis of variance on ranks with post hoc Tukey-Kramer's test. ${ }^{*} \mathrm{p}<0.01,{ }^{*} \mathrm{p}<0.05$. (B) Twenty-four hours after the final injection of ZOL solution, total RNA was purified from the tumors. Expression of mouse VEGF mRNA was analyzed by quantitative RT-PCR. Each result represents the mean \pm SD $(n=4)$.

In vitro antitumor effect. To confirm whether ZOL was taken up by tumor cells or macrophages, and was able to induce cytotoxic effects, we examined the cytotoxicity for LLC or RAW264.7 cells by ZOL. ZOL treatment showed higher cytotoxicity for RAW 264.7 cells than for LLC cells (Fig. 5A), indicating that this cytotoxicity by ZOL might be due to uptake by fluid-phase endocytosis in macrophage cells.

To examine the effect of ZOL on cytotoxicity by DXR, we examined the cytotoxicity for LLC or RAW264.7 cells by DXR in the presence of $20 \mu \mathrm{M} \mathrm{ZOL}$. ZOL showed additive cytotoxic effects for RAW 264.7 and LLC cells, rather than synergistic effects (Fig. 5B and C), suggesting that ZOL could not increase chemosensitivity by DXR for macrophages or LLC tumors.

Antitumor effect on LLC tumor-bearing mice. To examine whether ZOL injections could increase the antitumor effect of
Doxil by change of the tumor microenvironment, we evaluated the antitumor effect of Doxil after three intravenous injections of ZOL into LLC tumor-bearing mice. ZOL solution was intravenously administered on days 0,1 and 2, and then Doxil was on day 3. Three injections of ZOL solution did not show antitumor activity for the tumors (Fig. 6A), although they had an anti-angiogenic effect (Fig. 1). Single injection of Doxil showed a large antitumor effect. Furthermore, injections of ZOL increased the antitumor activity by Doxil. There were no remarkable differences in mouse body weight changes after the administration of ZOL and/or Doxil (Fig. 6B).

Accumulation of DXR liposomes in the tumor. Finally, we examined whether ZOL treatments affected the biodistribution of DXR in mice bearing LLC tumors after the injection of Doxil.ZOL injections significantly increased the blood concen- 

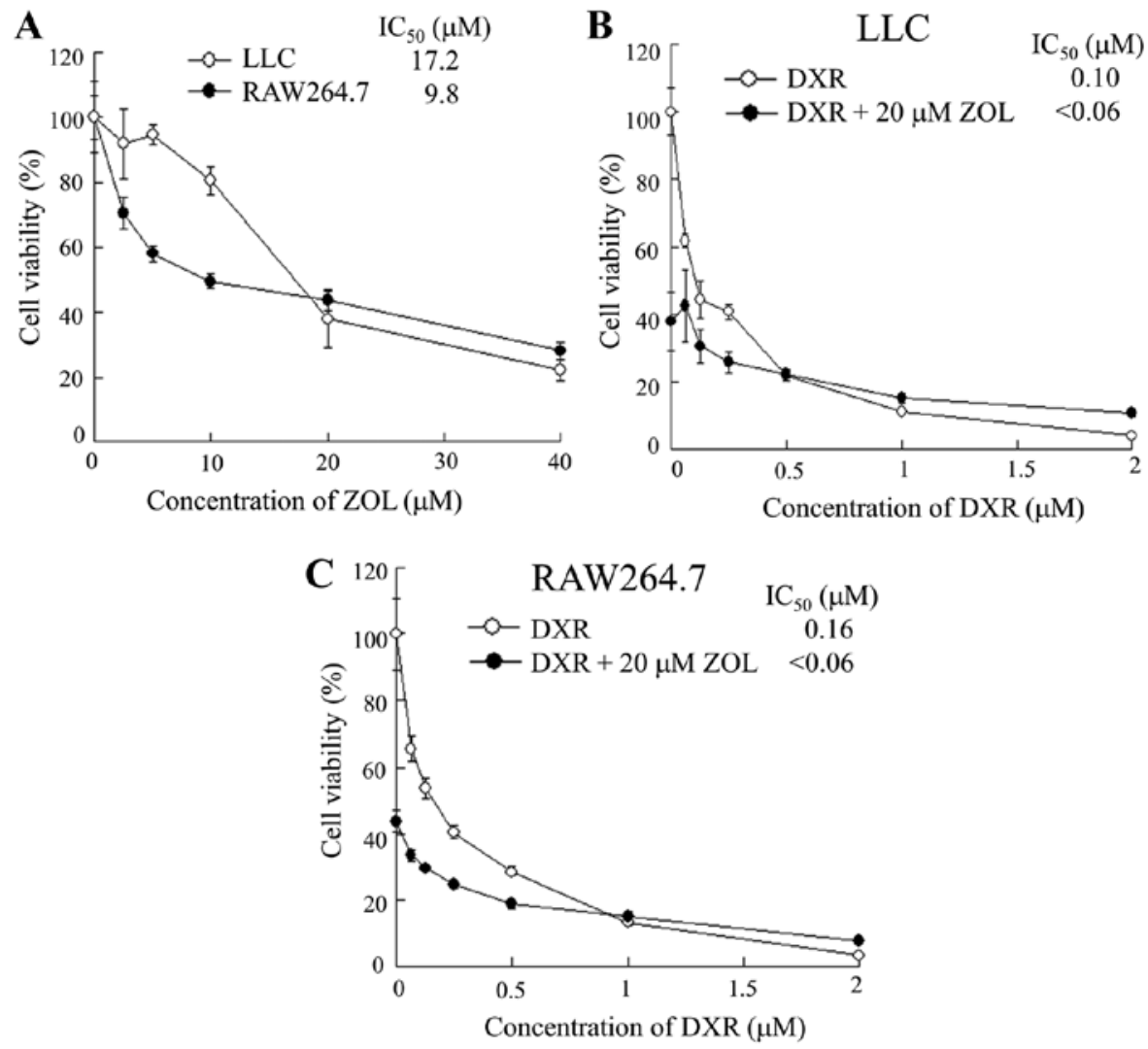

Figure 5. Cytotoxicity of ZOL for LLC and RAW264.7 cells (A) and of the combination of ZOL plus DXR for LLC (B) and RAW264.7 cells (C). (A) LLC and RAW264.7 cells were treated with various concentrations of ZOL for $48 \mathrm{~h}$. (B and C) LLC (B) and RAW264.7 cells (C) were treated with various concentrations of DXR in the presence or absence of $20 \mu \mathrm{M} \mathrm{ZOL}$ for $48 \mathrm{~h}$. Each result represents the mean $\pm \mathrm{SD}(\mathrm{n}=4)$.

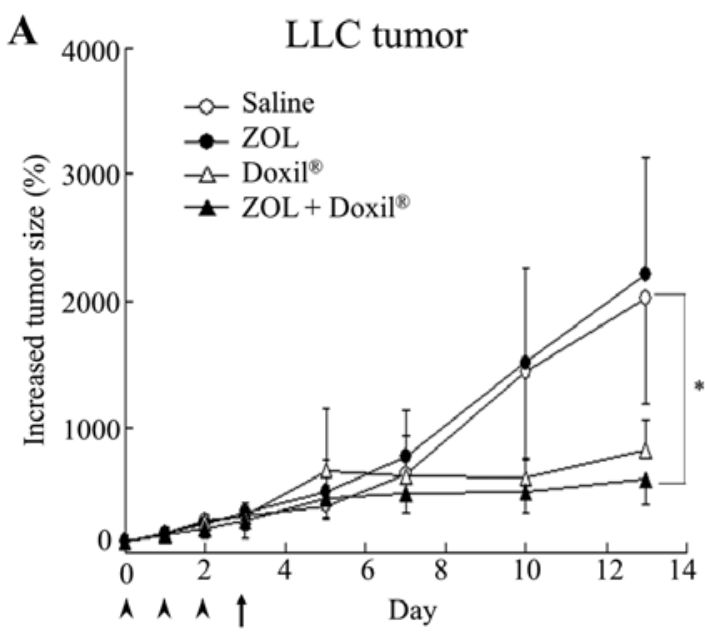

A $40 \mu \mathrm{g} \mathrm{ZOL} /$ mouse

$\uparrow 5 \mathrm{mg}$ DXR/kg (Doxil

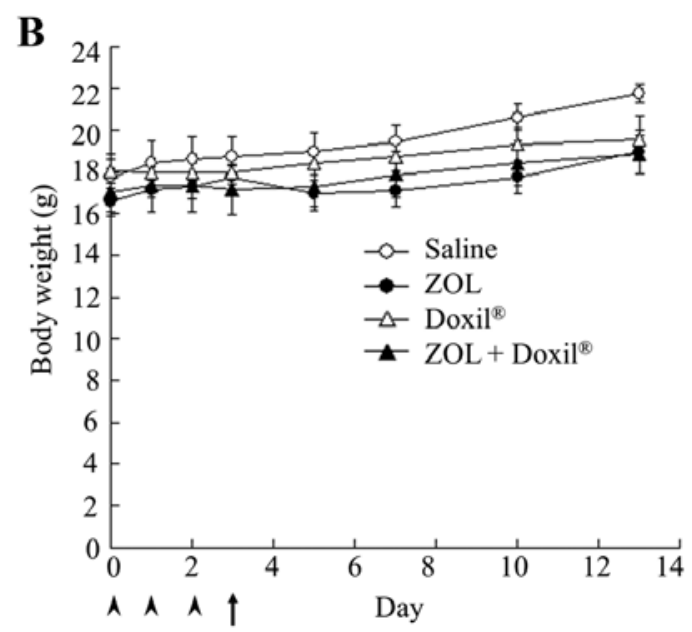

A $40 \mu \mathrm{g} \mathrm{ZOL/mouse}$

$\uparrow 5 \mathrm{mg} \mathrm{DXR} / \mathrm{kg}\left(\right.$ Doxil $\left.^{\mathbb{2}}\right)$

Figure 6. Combination therapy of ZOL and Doxil in LLC tumor-bearing mice. Antitumor activity and toxicity were assessed by measuring tumor volume (A) and body weight change (B). ZOL solution was administered on days 0,1 and 2 and Doxil was on day 3 . Arrowheads indicate the day of ZOL injection, and the arrow shows the day of Doxil injection. Each value represents the mean \pm SD $(n=3-4)$. The significance of differences between different groups was analyzed by one-way analysis of variance on ranks with post hoc Tukey-Kramer's test. "p<0.05.

tration of DXR after the injection of Doxil and decreased the accumulation of DXR in the liver (Fig. 7). The change of DXR accumulation in the liver may have been due to the depletion of Kupffer cells. However, the accumulation of DXR in the tumor was not significantly different between untreated and ZOL-treated tumors. These findings indicate that an increase of the antitumor effect of Doxil upon ZOL injections might be explained by an increased blood circulation time of Doxil 


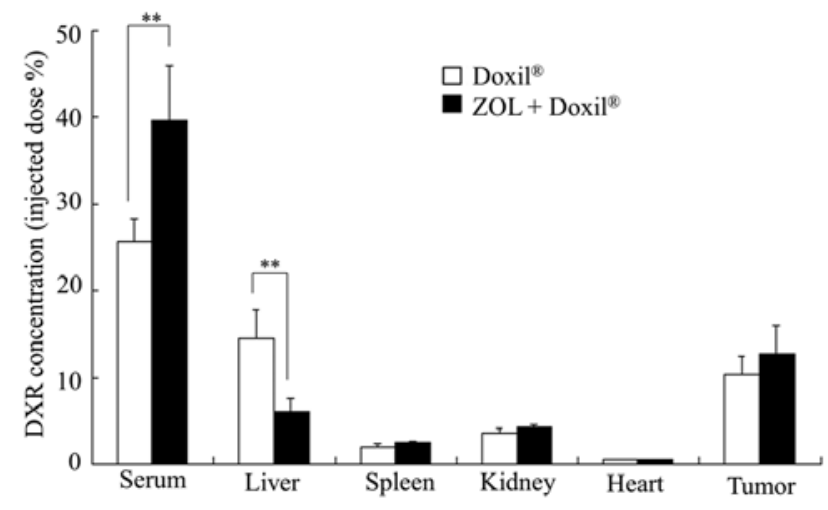

Figure 7. Effect of ZOL on the biodistribution of DXR at $24 \mathrm{~h}$ after the intravenous administration of Doxil into LLC tumor-bearing mice. ZOL solution was intravenously injected into tumor-bearing mice at a dose of $40 \mu \mathrm{g}$ of $\mathrm{ZOL} /$ mouse per day for three consecutive days. Twenty-four hours after the final injection of ZOL solution, Doxil was intravenously injected at $5 \mathrm{mg}$ of DXR $/ \mathrm{kg}$. DXR concentrations in serum, liver, spleen, kidney, heart and tumor were measured at $24 \mathrm{~h}$ after the injection of Doxil by HPLC. Each value represents the mean $\pm S D(n=3-4)$. Statistical significance was evaluated by Student's t-test. ${ }^{* *} \mathrm{p}<0.01$.

and/or wide distribution of DXR in the tumor by a change of the tumor microenvironment.

\section{Discussion}

Anti-angiogenesis effects are known to change the tumor vasculature. In this study, we found that ZOL treatment decreased IFP in tumor via the inhibition of tumor neovascularization (Figs. 1 and 2). Santini et al reported that single treatment of ZOL reduced circulating VEGF levels in cancer patients (17). However, in our study, a reduction of VEGF mRNA was not observed in tumors after ZOL treatments (Fig. 4B). Ogawara et al reported that VEGF did not play a major role in the angiogenesis in LLC tumors, suggesting that other proangiogenic factors except for VEGF might trigger angiogenesis in LLC tumors (18). Giraudo et al reported that ZOL suppressed the expression of matrix metalloproteinase-9 (MMP-9) by infiltrating macrophages and inhibited metalloproteinase activity, reducing the association of VEGF with its receptor on angiogenic endothelial cells (19). From these findings, the depletion of TAMs in the tumor by ZOL treatments might affect tumor neovascularization via inhibition of the association of VEGF and its receptor. However, it has also been reported that ZOL inhibited anti-angiogenesis through an apoptotic effect on endothelial cells in tumor and the tumor microenvironment $(20,21)$. ZOL exerts an inhibitory effect on endothelial cell adhesion and migration via the modulation of adhesion molecules (22). The mechanism by which ZOL treatments changed the vascular structures in the tumors was not clear, but ZOL treatments decreased IFP in the tumor via the inhibition of tumor neovascularization.

The most common adverse event associated with bisphosphonate therapy is transient fever (23). It has been shown that treatment with intravenous nitrogen-containing bisphosphonates such as ZOL caused systemic acute-phase responses (APRs) characterized by fever, pain, nausea and fatigue in up to $50 \%$ of all patients within $48 \mathrm{~h}$ after administration (24). These flu-like symptoms are typically transient, resolve spontaneously, and are accompanied by decreased lymphocyte counts and elevated levels of pro-inflammatory cytokines such as IL-6, IFN- $\gamma$ and TNF- $\alpha(23,25)$. In our study, we observed elevated levels of IL-10 and -12, GM-CSF and TNF- $\alpha$ after injections of ZOL solution into mice bearing a tumor (Fig. 4A); however, in normal mice without tumor, ZOL injections did not affect the level of inflammatory cytokines in serum. Although it was not clear why the ZOL treatments increased the levels of the inflammatory cytokines in tumor-bearing mice, these cytokines might be released from tumor tissues by ZOL treatments and cause inhibition of tumor neovascularization.

Polyethylene glycol (PEG)-modified liposomes are longlived in the circulation and accumulate in the tumors. The TGF- $\beta$ type I receptor inhibitors were reported to increase the antitumor effect of DXR encapsulated in PEGylated liposomes or micelles by changing the microenvironment of the vasculature $(12,13)$. Therefore, we examined whether ZOL treatments could increase the accumulation of Doxil in tumors and enhance the antitumor effect. As a result, ZOL treatments increased the antitumor effect of Doxil (Fig. 6); however, did not increase the accumulation of DXR in the tumor $24 \mathrm{~h}$ after the injection of Doxil (Fig. 7). ZOL is known as a specific inhibitor of farnesyl pyrophosphate synthase in the mevalonate pathway and exerts pleiotropic effects in tumor and non-tumor cells (26,27). Riganti et al reported that ZOL restored the chemosensitivity of DXR in multidrug-resistant cancer cells (28). However, in our study, ZOL treatments alone did not induce an antitumor effect in LLC tumors (Fig. 6), and did not show enhancement of cytotoxicity by DXR in LLC cells (Fig. 5B). Yoshizawa et al reported that pre-treatment with a VEGF receptor-2 inhibitor, SU5416, changed vascular structures in tumor but did not significantly increase the tumor accumulation of paclitaxel after the injection of PEGylated liposomal paclitaxel, compared with the untreated mice (29). However, they concluded that the treatment increased the distribution of PEGylated liposomal paclitaxel in the core region of the tumor, as well as conversely decreasing the ratio of its peripheral distribution. Therefore, we speculate that the enhanced antitumor effect observed in an in vivo experiment might be due to the improvement of DXR distribution in tumor, not an increase of DXR chemosensitivity in tumor cells. To prove this hypothesis, we observed the localizations of DXR in the tumor after ZOL treatments by fluorescent microscopy, but the localization was not well detected due to the weak intensity of DXR fluorescence (data not shown). Further study should be performed to investigate the distribution of DXR in tumor after ZOL treatments.

Resident macrophages in the liver called Kupffer cells comprise the major population of the reticuloendothelial system (RES). Doxil can avoid RES uptake by PEG modification; however, the effectiveness for the prevention of RES uptake is still incomplete. Previously, it was reported that the depletion of Kupffer cells by clodronic acid-entrapped liposomes (clodrolip) inhibited RES uptake in the liver and increased the plasma concentration of DXR after the injection of Doxil, resulting in enhancement of antitumor effects in a xenograft model (30). In our study, depletion of Kupffer cells (macrophages) in the liver was observed after the injection 
of ZOL (Fig. 3), and exhibited extended blood circulation of DXR and reduced its accumulation in the liver (Fig. 7). This depletion might be one of the reasons why the combination of ZOL and Doxil was able to enhance therapeutic efficacy.

Ottewell et al reported that the inhibition of tumor growth was observed by sequential injection with DXR and ZOL in a mouse model of breast and mammary tumor $(31,32)$. They concluded that sequential treatment with DXR followed by ZOL elicited substantial antitumor effects in vivo, but ZOL followed by DXR did not (31). The discrepancy between our results and previous reports might be caused by the schedule of administration of ZOL and DXR. In sequential treatment with ZOL followed by DXR, DXR was injected into the mice $24 \mathrm{~h}$ after the injection of ZOL; however, the tumors after ZOL treatment displayed no obvious differences in terms of the degree of vascularization compared with the saline control (31). In our experiments, no change of vascular structure in LLC tumors was observed $24 \mathrm{~h}$ after single injection of ZOL (Fig. 1A). These results might indicate that the repeated injections of $\mathrm{ZOL}$ were needed to increase the antitumor effect of DXR by the change of vascular structure.

In this study, we found that ZOL treatments decreased IFP in tumor via a change of tumor vasculature and enhanced the antitumor efficacy of liposomal doxorubicin (Doxil). ZOL treatment can be an alternative approach to increase the antitumor effect by liposomal drugs.

\section{Acknowledgements}

We thank Ms. Akira Kiyota for assistance in the experimental work. This study was supported in part by a Grant-in-Aid for Scientific Research (C) from the Japan Society for the Promotion of Science (KAKENHI grant no. 26460046).

\section{References}

1. Shang B, Cao Z and Zhou Q: Progress in tumor vascular normalization for anticancer therapy: Challenges and perspectives Front Med 6: 67-78, 2012.

2. Jain RK: Normalizing tumor vasculature with anti-angiogenic therapy: A new paradigm for combination therapy. Nat Med 7: 987-989, 2001.

3. Condeelis J and Pollard JW: Macrophages: Obligate partners for tumor cell migration, invasion, and metastasis. Cell 124: 263-266, 2006.

4. Pollard JW: Tumour-educated macrophages promote tumour progression and metastasis. Nat Rev Cancer 4: 71-78, 2004.

5. Kobayashi N, Miyoshi S, Mikami T, Koyama H, Kitazawa M, Takeoka M, Sano K, Amano J, Isogai Z, Niida S, et al: Hyaluronan deficiency in tumor stroma impairs macrophage trafficking and tumor neovascularization. Cancer Res 70: 7073- 7083, 2010.

6. Zhang W, Zhu XD, Sun HC, Xiong YQ, Zhuang PY, Xu HX, Kong LQ, Wang L, Wu WZ and Tang ZY: Depletion of tumorassociated macrophages enhances the effect of sorafenib in metastatic liver cancer models by antimetastatic and antiangiogenic effects. Clin Cancer Res 16: 3420-3430, 2010.

7. Rogers MJ, Gordon S, Benford HL, Coxon FP, Luckman SP, Monkkonen J and Frith JC: Cellular and molecular mechanisms of action of bisphosphonates. Cancer 88 (Suppl): 2961-2978, 2000.

8. Ross JR, Saunders Y, Edmonds PM, Patel S, Wonderling D, Normand $\mathrm{C}$ and Broadley K: A systematic review of the role of bisphosphonates in metastatic disease. Health Technol Assess 8: $1-176,2004$

9. Bäckman U, Svensson A, Christofferson RH and Azarbayjani F: The bisphosphonate, zoledronic acid reduces experimental neuroblastoma growth by interfering with tumor angiogenesis. Anticancer Res 28A: 1551-1557, 2008.
10. Goel S, Duda DG, Xu L, Munn LL, Boucher Y, Fukumura D and Jain RK: Normalization of the vasculature for treatment of cancer and other diseases. Physiol Rev 91: 1071-1121, 2011.

11. Hattori Y, Yamashita J, Sakaida C, Kawano K and Yonemochi E: Evaluation of antitumor effect of zoledronic acid entrapped in folate-linked liposome for targeting to tumor-associated macrophages. J Liposome Res: Sep 9, 2014 (Epub ahead of print). doi: 10.3109/08982104.2014.954128.

12. Kano MR, Bae Y, Iwata C, Morishita Y, Yashiro M, Oka M, Fujii T, Komuro A, Kiyono K, Kaminishi M, et al: Improvement of cancer-targeting therapy, using nanocarriers for intractable solid tumors by inhibition of TGF-beta signaling. Proc Natl Acad Sci USA 104: 3460-3465, 2007.

13. Taniguchi Y, Kawano K, Minowa T, Sugino T, Shimojo Y and Maitani Y: Enhanced antitumor efficacy of folate-linked liposomal doxorubicin with TGF- $\beta$ type I receptor inhibitor. Cancer Sci 101: 2207-2213, 2010.

14. Kato M, Hattori Y, Kubo M and Maitani Y: Collagenase-1 injection improved tumor distribution and gene expression of cationic lipoplex. Int J Pharm 423: 428-434, 2012.

15. Ogawara K, Un K, Minato K, Tanaka K, Higaki K and Kimura T: Determinants for in vivo anti-tumor effects of PEG liposomal doxorubicin: Importance of vascular permeability within tumors. Int J Pharm 359: 234-240, 2008.

16. Thompson K, Rogers MJ, Coxon FP and Crockett JC: Cytosolic entry of bisphosphonate drugs requires acidification of vesicles after fluid-phase endocytosis. Mol Pharmacol 69: 1624-1632, 2006.

17. Santini D1, Vincenzi B, Dicuonzo G, Avvisati G, Massacesi C, Battistoni F, Gavasci M, Rocci L, Tirindelli MC, Altomare V, et al: Zoledronic acid induces significant and long-lasting modifications of circulating angiogenic factors in cancer patients. Clin Cancer Res 9: 2893-2897, 2003.

18. Ogawara K, Abe S, Un K, Yoshizawa Y, Kimura T and Higaki K: Determinants for in vivo antitumor effect of angiogenesis inhibitor SU5416 formulated in PEGylated emulsion. J Pharm Sci 103: 2464-2469, 2014.

19. Giraudo $E$, Inoue $M$ and Hanahan D: An amino-bisphosphonate targets MMP-9-expressing macrophages and angiogenesis to impair cervical carcinogenesis. J Clin Invest 114: 623-633, 2004.

20. Wood J, Bonjean K, Ruetz S, Bellahcène A, Devy L, Foidart JM, Castronovo V and Green JR: Novel antiangiogenic effects of the bisphosphonate compound zoledronic acid. J Pharmacol Exp Ther 302: 1055-1061, 2002.

21. Corso A, Ferretti E and Lazzarino M: Zoledronic acid exerts its antitumor effect in multiple myeloma interfering with the bone marrow microenvironment. Hematology 10: 215-224, 2005.

22. Bezzi M, Hasmim M, Bieler G, Dormond O and Rüegg C: Zoledronate sensitizes endothelial cells to tumor necrosis factorinduced programmed cell death: Evidence for the suppression of sustained activation of focal adhesion kinase and protein kinase B/Akt. J Biol Chem 278: 43603-43614, 2003.

23. Dicuonzo G, Vincenzi B, Santini D, Avvisati G, Rocci L, Battistoni F, Gavasci M, Borzomati D, Coppola R and Tonini G: Fever after zoledronic acid administration is due to increase in TNF-alpha and IL-6. J Interferon Cytokine Res 23: 649-654, 2003.

24. Tanvetyanon T and Stiff PJ: Management of the adverse effects associated with intravenous bisphosphonates. Ann Oncol 17: 897-907, 2006.

25. Reid IR, Gamble GD, Mesenbrink P, Lakatos P and Black DM: Characterization of and risk factors for the acute-phase response after zoledronic acid. J Clin Endocrinol Metab 95: 4380-4387, 2010.

26. Clézardin P and Massaia M: Nitrogen-containing bisphosphonates and cancer immunotherapy. Curr Pharm Des 16: 3007-2014, 2010.

27. Coscia M, Quaglino E, Iezzi M, Curcio C, Pantaleoni F, Riganti C, Holen I, Mönkkönen H, Boccadoro M, Forni G, et al: Zoledronic acid repolarizes tumour-associated macrophages and inhibits mammary carcinogenesis by targeting the mevalonate pathway. J Cell Mol Med 14: 2803-2815, 2010.

28. Riganti C, Castella B, Kopecka J, Campia I, Coscia M, Pescarmona G, Bosia A, Ghigo D and Massaia M: Zoledronic acid restores doxorubicin chemosensitivity and immunogenic cell death in multidrug-resistant human cancer cells. PLoS One 8: e60975, 2013. 
29. Yoshizawa Y, Ogawara K, Fushimi A, Abe S, Ishikawa K, Araki T, Molema G, Kimura T and Higaki K: Deeper penetration into tumor tissues and enhanced in vivo antitumor activity of liposomal paclitaxel by pretreatment with angiogenesis inhibitor SU5416. Mol Pharm 9: 3486-3494, 2012.

30. Ohara Y, Oda T, Yamada K, Hashimoto S, Akashi Y, Miyamoto R, Kobayashi A, Fukunaga K, Sasaki R and Ohkohchi N: Effective delivery of chemotherapeutic nanoparticles by depleting host Kupffer cells. Int J Cancer 131: 2402-2410, 2012.
31. Ottewell PD, Mönkkönen H, Jones M, Lefley DV, Coleman RE and Holen I: Antitumor effects of doxorubicin followed by zoledronic acid in a mouse model of breast cancer. J Natl Cancer Inst 100: 1167-1178, 2008.

32. Ottewell PD, Brown HK, Jones M, Rogers TL, Cross SS, Brown NJ, Coleman RE and Holen I: Combination therapy inhibits development and progression of mammary tumours in immunocompetent mice. Breast Cancer Res Treat 133: 523-536, 2012. 